\title{
EVALUATION OF POLARIMETRIC FEATURES FOR SEA ICE CHARACTERIZATION AT X, C AND L- BAND SAR
}

\author{
Suman Singha; \\ Maritime Safety and Security Lab \\ Remote Sensing Technology Institute (IMF), German Aerospace Center (DLR) \\ Heinrich Focke Str. 4, 28199, Bremen, Germany \\ Suman.Singha@dlr.de
}

\begin{abstract}
In recent years SAR Polarimetry has become a valuable tool in space-borne SAR based sea ice analysis. This work compares the polarimetric backscatter behavior of sea ice in space-borne X-band C-band and L-band Synthetic Aperture Radar (SAR) imagery. Two sets of spatially and temporally near coincident fully polarimetric acquisitions from the TerraSAR-X/TanDEM-X, RADARSAT-2 and ALOS-2 satellites are investigated. Our algorithmic approach for an automated sea ice classification consists of two steps. In the first step, we perform a polarimetric feature extraction procedure. The resulting feature vectors are then ingested into a trained neural network classifier to arrive at a pixel-wise supervised classification. Based on the common coherency and covariance matrix, we extract a number of features and analyze the relevance and redundancy by means of mutual information for the purpose of sea ice classification. Coherency matrix based features which require an eigendecomposition are found to be either of low relevance or redundant to other covariance matrix based features, which makes coherency matrix based features dispensable for the purpose of sea ice classification. Among the most useful features for classification are matrix invariant based features (Geometric Intensity, Scattering Diversity, Surface Scattering Fraction). This analysis reveals analogous results for all four acquisitions, in both X-band and C-band frequencies and slightly different for L-band. The subsequent classification produces similarly promising results for all four acquisitions. In particular, the overlapping image portions exhibit a reasonable congruence of detected
\end{abstract}

Index Terms - Cyrosphere, Pol-SAR, Sea ice, NRT Processing, Artificial Neural Network, TerraSAR-X, RADARSAT2, ALOS-2.

TerraSAR-X Images were acquired through Science AO OCE 2985 (DRA). RADARSAT-2 Quad Pol Images were acquired through ESA Third Party Mission. ALOS-2 dataset were kindly provided by JAXA.

\section{INTRODUCTION}

Spaceborne SAR data has become a powerful means for sea ice monitoring since the launch of the first spaceborne platforms some decades ago (see eg. [1, 2]). The two major objectives in SAR based remote sensing of sea ice is on the one hand to have a large coverage of the imaged ground area, and on the other hand to obtain a radar response that carries as much information as possible. Whereas single-polarimetric acquisitions of existing sensors offer a wide coverage on the ground, dual polarimetric, or even better fully polarimetric data offer a higher information content which allows for a more reliable automated sea ice analysis. A common approach in automated sea ice classification based on SAR imagery is to first compute a vector of features pixelwise or for small neighborhoods of the image. While single-pol data can only be analyzed by classical image analysis for one channel (e.g. texture analysis), complex dual or fully polarimetric data allows the application of polarimetric analysis. After the feature extraction, one then has to choose a classification approach. Our goal is to achieve a maximal degree of automation in our process chain, wherefore we adopt a supervised classification technique so we can include expert knowledge in our process chain. More precisely, a popular neural network library (FANN) is built into our algorithm. For operational purposes, the classifier needs to take into account variations due to region, season and in particular for different incidence angle ranges, when training according to expert information (in-situ observations, official ice charts): SAR images where ice classes are known with reasonable certainty serve as templates so the pertaining feature information from these images can be used train the classifier function to generate the reference output. Further details of such an neural network approach can be found in [1],[3] or [4].

\section{DATASET}

The underlying datasets were acquired with high temporal and spatial correlation in X, C and L-band. In this work 
Table 1. TerraSAR-X and RADARSAT-2 imaging modes used in this study. * DRA - Dual Receive Antenna (Experimental Mode), SM - StripMap, FQ - Fine Quad, HBQ - High-sensitive Quad Q - Quadpol

\begin{tabular}{c|c|c|c|c|c|c} 
Date, time (UTC) & Sensor & $\begin{array}{c}\text { Inc. } \\
\text { Angle. }\end{array}$ & $\begin{array}{c}\text { Polar. } \\
\text { Mode }\end{array}$ & $\begin{array}{c}\text { Imaging } \\
\text { Mode }\end{array}$ & $\begin{array}{c}\text { Slant Rg./ } \\
\text { Az. Res.(m) }\end{array}$ & $\begin{array}{c}\text { Footprint } \\
\text { Az } \times \text { Rg. }\end{array}$ \\
\hline \hline $2015 / 04 / 19,14: 51$ & TS-X & $39.73^{\circ}$ & Q* $^{*}$ & SM & $1.2 \mathrm{~m} / 6 \mathrm{~m}$ & $115 \mathrm{~km} \times 17.5 \mathrm{~km}$ \\
\hline $2015 / 04 / 19,13: 19$ & RS-2 & $21.78^{\circ}$ & Q & FQ & $5.2 \mathrm{~m} / 7.6 \mathrm{~m}$ & $75 \mathrm{~km} \times 27 \mathrm{~km}$ \\
\hline $2015 / 04 / 19,20: 32$ & ALOS-2 & $33.90^{\circ}$ & Q & HBQ & $5.1 \mathrm{~m} / 4.3 \mathrm{~m}$ & $70 \mathrm{~km} \times 42 \mathrm{~km}$ \\
\hline $2015 / 04 / 23,13: 43$ & TS-X & $27.20^{\circ}$ & $\mathrm{Q}^{*}$ & SM & $1.2 \mathrm{~m} / 6 \mathrm{~m}$ & $150 \mathrm{~km} \times 17.5 \mathrm{~km}$ \\
\hline $2015 / 04 / 23,14: 42$ & RS-2 & $37.25^{\circ}$ & Q & FQ & $5.2 \mathrm{~m} / 7.6 \mathrm{~m}$ & $52.5 \mathrm{~km} \times 27 \mathrm{~km}$ \\
\hline $2015 / 04 / 23,20: 18$ & ALOS-2 & $33.90^{\circ}$ & Q & HBQ & $5.1 \mathrm{~m} / 4.3 \mathrm{~m}$ & $70 \mathrm{~km} \times 42 \mathrm{~km}$
\end{tabular}

we want to explore sea ice classification on full-polarimetric data with secondary priority given to resolution, which naturally comes at the price of a smaller footprint. A list of the datatakes with the respective technical details can be found in $[5,1]$ (and figures therein). We remark that each acquisition consists of two or three frames (frame corresponds to nominal acquisition length in azimuth direction). The images were acquired north of Svalbard between the latitudes 82.4 and 83.4 North and longitudes 11 and 23 East, in the Arctic Ocean. Precise coordinates will be displayed in 2 .

Training data rectangles in the image were determined by visual judgement in conjunction with archive data of usual ice situation for the location and time of the year. The reported average ice thickness for the region of the training dataset was above $0.90 \mathrm{~m}$ according to L3C SMOS data (icdc.zmaw.de/daten/cryosphere/13c-smos-sit.html) on $2015 / 04 / 15$. Ice concentration charts of the Norwegian ice service reported a local average sea ice concentration of $100 \%$. According to NASA MODIS data on $2015 / 04 / 19$ and 2015/04/23 (OB.DAAC, [1]), the sea surface temperature in the region of datatakes is below $-10^{\circ}$ Celsius (apart from open water portions), wherefore we conclude that thaw onset can be has not occurred for our dataset. The regime of dominant ice classes found were open water (OW), young ice (YI), smooth first year (SFYI) ice and a mixture of rough first-year and multi-year ice (RFYMYI).

\section{ASSESSMENT OF POLARIMETRIC FEATURES}

To quantify the information content rigorously, we use the concept of mutual information from information theory, which has become a rather prominent tool in the investigation of information content and discriminative power ( $c f$. [6]). A detailed description of methodology and analysis on the polarimetric features can be found in [1] for this particular dataset (except L-band acquisitions). To sum up the essence of our relevance and redundancy results: For TS-X, RS-2 and ALOS-2 acquisitions we found similarly high relevance for a number of lexicographic features and likewise rather low relevance for Pauli based features (Fig. 1). Additionally, when a Pauli feature exhibited at least mediocre relevance, namely $H^{(q)}$, it was found to be closely (mutual-information)correlated to another lexicographic feature, namely $\delta$. This renders $H^{(q)}$ dispensable with high likelihood. For deep mathematical reasons, this close information relationship between $H^{(q)}$ and $\delta$ is true in general and not just for our particular datasets ([1]). In case of ALOS-2 acquisitions we found slightly deferent behavior of polarimetric features where Correlation $(\varepsilon)$ and Real part of the copolarization cross product $(\rho)$ performed significantly better compared to $\mathrm{X}$ and $\mathrm{C}$ band acquisitions Such a finding about the suitability of features for sea ice classification can then be used to justify discarding some features in the processing chain.

\section{CLASSIFICATION RESULTS}

As mentioned in the introduction, we performed a pixelwise supervised classification using a neural network. The feature set we used contained all features plus their respective local variances, which was computed for each center pixel of a 11 $\times 11$ submatrix sliding over the entire feature image. Thus, we extracted for each pixel outlined polarimetric features and then ingested the feature vectors into the classifier. The implementation was carried out in the Exelis IDL programming language (image ingestion, calibration, feature extraction, statistical analysis) and in C (FANN library classifier). The hardware specifications used were: 11 GB RAM, Intel Core i-7 $3740 \mathrm{QM}$, virtual linux OS. The processing time was $20 \mathrm{~min}$ in total for feature extraction and classification. In order to validate the stability of the training process, we randomly split the initial training data patches into two disjoint subsets and generated 10 different classifiers. The classification results compared to reference data samples (as presented in Table 2) exhibit a very promising accuracy (using averaged accuracies over different classifiers), which underscores the stability of our algorithm. The percentages in the matrix indicate the proportion of samples of one reference class that were assigned to the respective ice type by the classifier. Therefore columns add up to $100 \%$. The test was carried out for all acquisitions with variations of less than $4 \%$ in the following accuracy matrix. We therefore only include the results for the ALOS-2 acquisition of 23 April 2015.

The distinction of all classes of ice, specially Open Water 

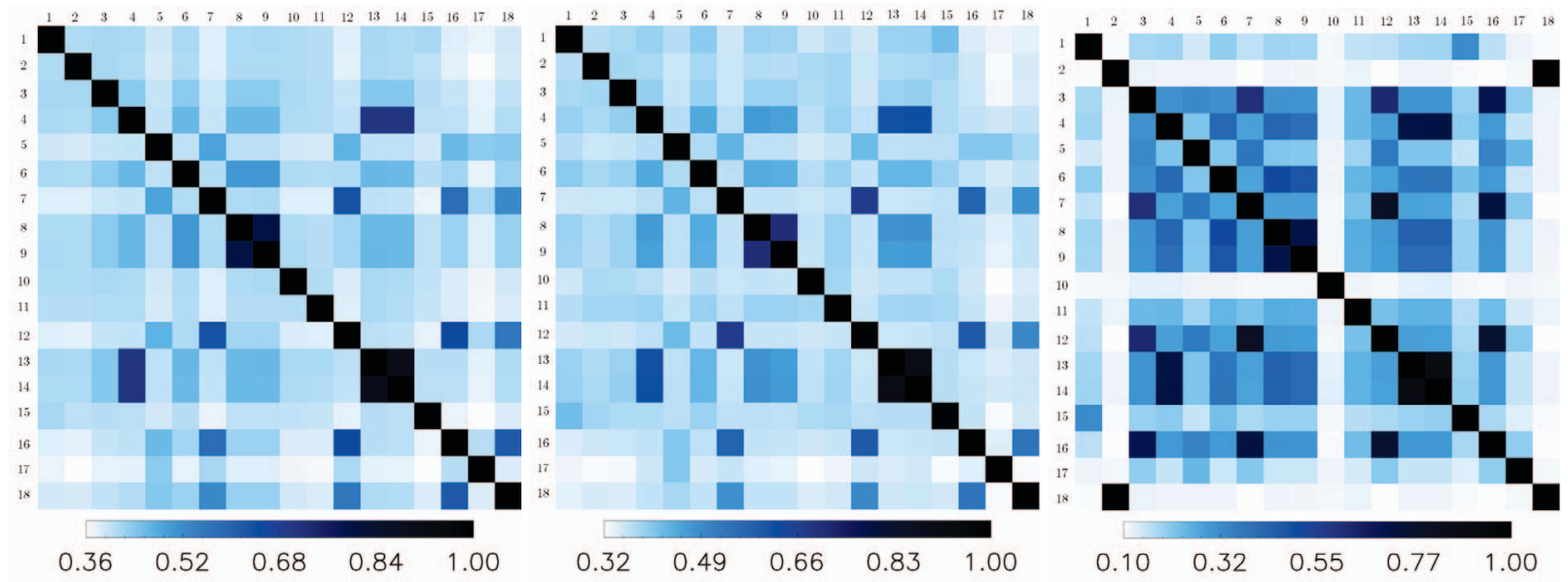

Fig. 1. Normalized mutual information for TS-X (Left), RS-2 (Middle) and ALOS-2 (Right) acquisition of 23 April 2015, $\mathcal{I}\left(Y_{1}, Y_{2}\right) / \sqrt{\mathcal{H}\left(Y_{1}\right) \mathcal{H}\left(Y_{2}\right)}$. Features are: 1: $\gamma, 2: \Delta \phi, 3: \rho, 4: \varepsilon, 5: \mu, 6: \tau, 7: \operatorname{span}^{(q)}, 8: \delta, 9: H^{(q)}, 10: A^{(q)}, 11: \alpha^{(q)}$, 12: $\operatorname{span}^{(d)}, 13: H^{(d)}, 14: A^{(d)}, 15: \alpha^{(d)}, 16: \lambda_{1}^{(d)}, 17: \lambda_{2}^{(d)}, 18: \operatorname{Var} \Delta \phi$.
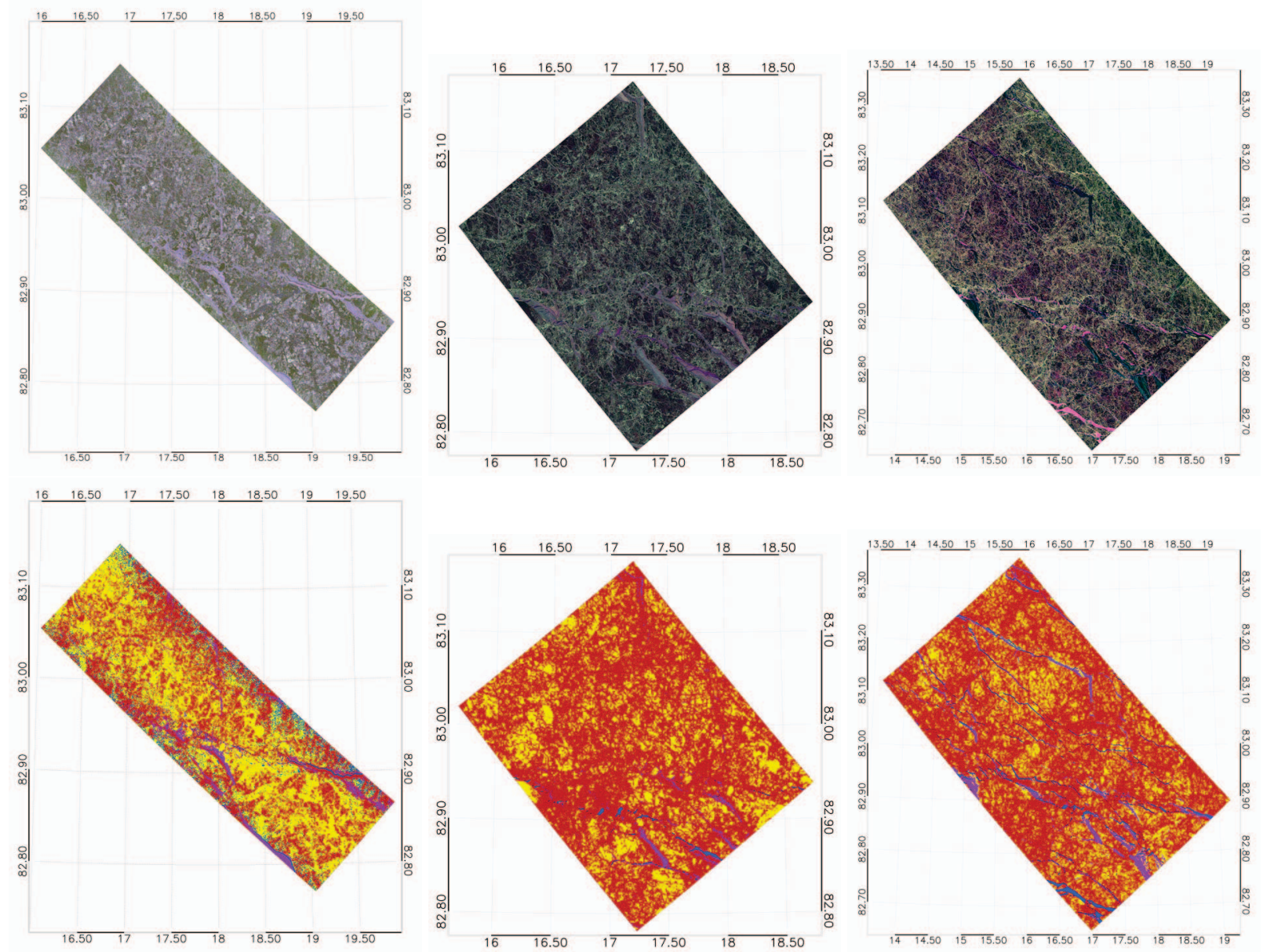

Fig. 2. Top: Geocoded Pauli RGB composite (Red: HH-VV Green: HV+VH Blue: HH+VV) of the TS-X (Left), RS2(Middle) and ALOS-2 (Right) acquisition on 23 April 2015; Bottom: ice classification on TS-X (Left), RS-2 (Middle) and ALOS-2 (Right) acquisition. Blue: open water/nilas (OW),purple: young ice (YI), yellow: first year ice (SFYI), red: rough first year ice/multi-year ice (RFYMYI). 
Table 2. Classification results compared to reference data samples from each class, averaged over different neural network topologies, ALOS-2 acquisition 23 April 2015.

\begin{tabular}{|c||c|c|c|c|}
\hline \multicolumn{1}{|c||}{} & \multicolumn{4}{c|}{ Reference ice class } \\
\hline \hline ANN Result & OW & YI & SFYI & RFYMYI \\
\hline OW & $100.0 \%$ & $\%$ & $0 \%$ & $0 \%$ \\
\hline YI & $0 \%$ & $97.3 \%$ & $0 \%$ & $0 \%$ \\
\hline SFYI & $0 \%$ & $0 \%$ & $96.9 \%$ & $3.0 \%$ \\
\hline RFYMYI & $0 \%$ & $2.7 \%$ & $3.1 \%$ & $97.0 \%$ \\
\hline
\end{tabular}

and Young Ice are quite promising (see Table 2 ). Noting that both training and validation data are from the same ice situation (i.e., same time, location and incidence angle), our approach can be considered to be consistent in itself and stable in terms of the choice of the training data. When the ice situation does not vary significantly, our method can be expected to produce very reasonable results. To visually assess the classification, we juxtaposed TS-X, RS-2 and ALOS-2 Pauli RGB and Classified results in Figure 2.

We notice in the TS-X image of 23 April 2015 that RFYMYI tends to be overstated in the classification near the vertical margins. Such tendencies we do not observe in the TS-X image with higher incidence angle, nor in any of the RS-2 and ALSO-2 images. So we summarize that for the locations of recognizably identical ice structures, we deem it justified to observe significant general match in the classified ice types with a possible incidence angle induced bias for certain ice types. This bias we observe similarly for X-band and C-band and slightly less for the L-band. The noticeable noise pattern (especially on the vertical margins) of the TS-X near range image of 23 April 2015 suggests the preferred use higher incidence angle acquisitions (i.e., above $30^{\circ}$ ). Such incidence angle biases certainly need to be addressed when establishing the classifier. This also stresses the necessity to establish a library of robust classifiers for different incidence angle ranges.

\section{CONCLUSIONS}

In this work, we conducted a thorough, quantitative comparison of spatially and temporarily near coincident quadpolarimetric images in X-band and C-band in terms of feature suitability for sea ice classification. In order to judge in a rigorous and quantitative way on the suitability of different polarimetric features, we performed a mutual information based analysis of the data from all four acquisitions. Based on this analysis we arrived at the conclusion that, for our purposes, features involving eigendecomposition of the scatter coherency matrix $T_{3}$ do not provide informational benefit over features purely based on the covariance matrix $S_{3}$. Given the spatial and time correlation, in our findings we can preclude any impact of ice scene variability. The findings about the relevance and redundancy of particular features turned out to be similar for either of the SAR frequency bands. All extracted features were then used to train a neural network classifier. Numerical and visual analysis of results are rather promising in terms of providing inputs into the creation of operational ice charts. Future efforts will be devoted to refining the proposed methodology and testing our automated sea ice classification algorithm on pruned feature sets, in particular omitting eigendecomposition based features, excluding phase related information, or working with HH-VV information only. The generalizability and limitations of our method will be tested on a more extensive dataset and compared with ground truth collected during N-ICE 2015 Campaign.

\section{REFERENCES}

[1] R. Ressel and S. Singha, "Comparing near coincident space borne $\mathrm{C}$ and $\mathrm{X}$ band fully polarimetric sar data for arctic sea ice classification," Remote Sensing, vol. 8, no. 3, p. 198, 2016. [Online]. Available: http://www.mdpi.com/2072-4292/8/3/198

[2] M. A. N. Moen, A. P. Doulgeris, S. N. Anfinsen, A. H. H. Renner, N. Hughes, S. Gerland, and T. Eltoft, "Comparison of feature based segmentation of full polarimetric SAR satellite sea ice images with manually drawn ice charts," The Cryosphere, vol. 7, no. 6, pp. 1693-1705, 2013. [Online]. Available: http://www.the-cryosphere.net/7/1693/2013

[3] R. Ressel, S. Singha, S. Lehner, A. Rsel, and G. Spreen, "Investigation into different polarimetric features for sea ice classification using x-band synthetic aperture radar," IEEE Journal of Selected Topics in Applied Earth Observations and Remote Sensing, vol. 9, no. 7, pp. 3131-3143, July 2016.

[4] N. Y. Zakhvatkina, V. Alexandrov, O. M. Johannessen, S. Sandven, and I. Frolov, "Classification of sea ice types in ENVISAT synthetic aperture radar images," IEEE Transactions on Geoscience and Remote Sensing, vol. 51, pp. 2587-2600, 2013.

[5] A. Johansson, J. King, A. Doulgeris, S. Gerland, S. Singha, G. Spreen, and T. Busche, "Combined observations of arctic sea ice with near-coincident colocated x, c, and l-band sar satellite remote sensing and helicopter-borne measurements," Journal of Geophysical Research: Oceans, pp. n/a-n/a, 2016. [Online]. Available: http://dx.doi.org/10.1002/2016JC012273

[6] H. Peng, F. Long, and C. Ding, "Feature selection based on mutual information criteria of max-dependency, maxrelevance, and min-redundancy," Pattern Analysis and Machine Intelligence, IEEE Transactions on, vol. 27, no. 8, pp. 1226-1238, Aug 2005. 